\title{
Iterative Image Reconstruction for Emission Tomography using Fuzzy Potential
}

\author{
Partha P. Mondal \\ Department of Physics \\ Indian Institute of Science \\ Bangalore, India \\ Email: partha@physics.iisc.ernet.in
}

\author{
K. Rajan \\ Department of Physics \\ Indian Institute of Science \\ Bangalore, India \\ Email: partha@physics.iisc.ernet.in
}

\begin{abstract}
The Maximum a-posteriori (MAP) and maximum likelihood (ML) algorithm produces good reconstruction for emission tomography. However they still suffer from noise and optimal smoothing. Penalized iterative algorithms based on MAPestimation often result in over smooth reconstructions. These algorithms fail to determine the density class in the reconstructed image and hence penalize the pixels irrespective of the density class. Reconstruction with better edge information is often difficult due to the lack of prior knowledge. In this paper, a fuzzy logic based approach is proposed to model the nature of pixel-pixel interaction. The proposed algorithm consists of two elementary steps: (1) Edge detection - fuzzy rule based derivatives are used for the detection of edges in the nearest neighborhood window. (2) Fuzzy smoothing - penalization is performed only for those pixels for which no edge is detected in the nearest neighborhood. Both of these operations are carried out iteratively until convergence. Quantitative analysis shows that the proposed fuzzy rule based reconstruction algorithm is capable of producing better reconstructed images when compared with MAP and MRP reconstructed images.
\end{abstract}

\section{INTRODUCTION}

algorithms are central to many image reconstruction problems. Medical diagnostic imaging modalities like positron emission tomography (PET), single photon emission computed tomography (SPECT) demand high quality images. Though iterative algorithms like, maximum likelihood (ML) [1], maximum a-posteriori (MAP) [2][3][4] and median root prior (MRP) [5][6] algorithms are capable of generating good quality images in ET, but at the cost of artifacts like noise, over-smoothness, streaking effect [5] and hence require improvement.

In the present work, fuzzy techniques have been successfully applied for image reconstruction in ET. Prior distribution is defined by Gibbs distribution and the potential (which defines the nature of nearest neighbor interactions), and is modeled using fuzzy rules.

\section{IMAge RECONStruction ALgORIthMs FOR PET}

The measurements in PET , $y_{j}, j=1, \ldots, \mathrm{M}$ are modeled as independent Poisson random variables i.e, $y_{j} \sim \operatorname{Poisson}\left(\sum_{i=1}^{N} \lambda_{i} p_{i j}\right)$ for $j=1, \ldots, \mathrm{M}$, where $\lambda_{i}$, $i=1, \ldots, \mathrm{N}$ are the mean parameters of the emission process and $p_{i j}$ is the probability that an annihilation in the $i^{t h}$ pixel is detected in $j^{\text {th }}$ detector. The likelihood function is given by,

$$
P(y / \lambda)=\prod_{j=1}^{M}\left[\frac{\exp \left(-\sum_{i=1}^{N} \lambda_{i} p_{i j}\right)\left(\sum_{i=1}^{N} \lambda_{i} p_{i j}\right)^{y_{j}}}{y_{j} !}\right]
$$

The image field is assumed to be a Markov random field (MRF) [3] and by Hammerseley-Clifford theorem [7], image $\lambda$ is characterized by Gibbs distribution,

$$
P(\lambda)=\frac{1}{Z} e^{-\frac{1}{\beta} \sum_{i} \sum_{j \in N_{i}} w_{i j} V\left(\lambda_{i}, \lambda_{j}\right)}
$$

where, $\mathrm{Z}$ is the normalizing constant for the distribution, $\beta$ is the Gibbs hyper-parameter, $w_{i j}$ is the weight of pixel $j \epsilon N_{i}$ [2], $N_{i}$ is the nearest neighbor set of pixel $i$ and $V\left(\lambda_{i}, \lambda_{j}\right)$ is termed as the potential at site $i$ due to the nearest neighbor elements $j$.

MAP algorithm determines that estimate $\lambda^{M A P}$ as the solution which maximizes the posterior density function $P(\lambda / y)$ or equivalently the $\log$ of $P(\lambda / y)$. Given a suitable prior $P(\lambda)$, MAP-reconstruction can be formulated as,

$$
\lambda^{M A P}=\max _{\lambda \geq 0}[\log P(y / \lambda)+\log P(\lambda)]
$$

Solution for eqn.(4) is very difficult due to the complicated nature of the prior. Green [2] has proposed one step late (OSL) approximation for an iterative update to the MAP-problem and is given by,

$$
\lambda_{i}^{k+1}=\frac{\lambda_{i}^{k}}{\left[\sum_{j=1}^{M} p_{i j}+\frac{1}{\beta} \sum_{j \epsilon N_{i}} w_{i j}\left(\frac{\partial V\left(\lambda_{i}, \lambda_{j}\right)}{\partial \lambda_{i}}\right)_{\lambda_{i}=\lambda_{i}^{k}}\right]} \times
$$

$$
\sum_{j=1}^{M} \frac{y_{j} p_{i j}}{\sum_{i=1}^{N} \lambda_{i}^{k} p_{i j}}
$$

Given the iterative OSL-algorithm (eqn.(6)), the next step is the proper modeling of the interacting potential $V\left(\lambda_{i}, \lambda_{j}\right)$ between the pixel at site $i$ and its neighbors $j \in N_{i}$. 

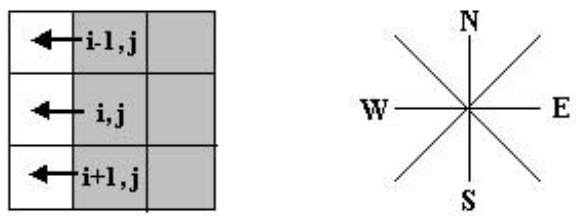

Fig. 1. $3 \times 3$ neighborhood of a central pixel $(i, j)$, showing the directional derivative along $\hat{W}$.

\section{FuZzy Logic Based Potential Function}

A fuzzy logic based potential is proposed for edgepreserving PET reconstruction. This consists of two basic operations ; fuzzy filtering and fuzzy smoothing. The proposed fuzzy logic is similar to that used by Ville et. al. for image restoration [8]. Nevertheless the idea is expanded, moulded and adapted for ET (PET, SPECT).

The first derivatives $\nabla^{k}(i, j)$ for the pixel centered at $(i, j)$, along the direction $\hat{n}$ at $k^{t h}$ iteration is defined as,

$$
\nabla^{k}(i, j) \hat{n}=\left|\lambda^{k}(i, j)-\lambda^{k}(*, *)\right| \hat{n}
$$

where, $\lambda^{k}(*, *) \hat{n}$ represents the nearest pixel value along the unit directional vector $\hat{n}$. For example,

$$
\nabla^{k}(i, j) \hat{W}=\left|\lambda^{k}(i, j)-\lambda^{k}(i, j-1)\right| \hat{W}
$$

For identifying an edge along a particular direction, three derivatives are chosen (see Fig. 1). For example, to detect an edge along N-S direction, the derivatives are: $\nabla^{k}(i, j) \hat{W}$, $\nabla^{k}(i-1, j) \hat{W}$ and $\nabla^{k}(i+1, j) \hat{W}$ for a $3 \times 3$ window. The values of the derivatives will be large if there is an edge along $\mathrm{N}-\mathrm{S}$ direction. It is safe to assume that if two out of three derivatives are small, an edge is absent in the neighborhood. This is termed as 2:3 rule. The membership function for the property small is defined in the next subsection. To compute the value that expresses the degree to which the fuzzy derivative in a certain direction is small, we will make use of the fuzzy set small. For $\hat{n}=\hat{W}$ the fuzzy derivative is defined as follows:

if

$$
\left\{\begin{array}{l}
\nabla^{k}(i, j) \text { and } \nabla^{k}(i-1, j) \text { are small or } \\
\nabla^{k}(i, j) \text { and } \nabla^{k}(i+1, j) \text { are small or } \\
\nabla^{k}(i-1, j) \text { and } \nabla^{k}(i+1, j) \text { are small }
\end{array}\right.
$$

then, $\nabla_{F}^{k}(i, j)$ is small,

$$
\text { else, } \nabla_{F}^{k}(i, j) \text { is large }
$$

Similarly, the values of the fuzzy derivatives $\nabla_{F}^{k}(i, j) \hat{n}$ for all the directions i.e, $\{\hat{E}, \hat{W}, \hat{N}, \hat{S}, \hat{N E}, N \hat{W}, \hat{S E}, S \hat{W}\}$ are calculated.

Fuzzy sets are best represented by membership function. Membership function gives the degree of belongingness within the set. The membership function $\mu$ of a fuzzy set $X$, maps the elements of $X$ into a numerical value $\{0,1\}$ i.e, $X \stackrel{\mu}{\rightarrow}\{0,1\}$. A membership function similar to Zadeh's S-function [9] has been used for modeling the property small. The proposed membership function for the property small of the pixel $\lambda^{k}(i, j)$ at $k^{t h}$ iteration is defined as,

$$
\mu^{k}(i, j)= \begin{cases}2\left[\frac{\nabla-a}{c-a}\right]^{2}, & a \leq \nabla \leq b \\ 1-2\left[\frac{\nabla-c}{c-a}\right]^{2}, & b<\nabla \leq c\end{cases}
$$

where,

$$
\begin{gathered}
a=\min (\nabla) \\
b=\nabla_{M}=\frac{1}{8} \sum_{\hat{n}} \nabla \hat{n} \\
c=\max (\nabla)
\end{gathered}
$$

For brevity, we have denoted $\nabla^{k}(i, j)$ by $\nabla$ and $\nabla_{M}^{k}(i, j)$ by $\nabla_{M} . a$ and $b$ respectively are the minimum and maximum value of the derivative $\nabla$ in the neighborhood window centered at $(i, j) . \nabla_{M}$ represents the mean variation of the gray level values in the nearest neighborhood window centered at $(i, j)$. Since we are looking for edges in an $s \times s$ neighborhood window, we have taken the mean of the gray level differences for edge detection. Presence of edge inside the window produces large $\nabla$, where as, absence of edge would result in small $\nabla$. It is assumed that an edge is present if $\nabla_{F}>b$, otherwise edge is absent. These values are calculated separately for each pixel and get updated iteratively, thereby determining the shape of membership function small. Within this framework, fuzzy set small corresponds to the values in the range [a,b] with a membership value $\left\{0 \leq \mu \leq \mu_{b}\right\}$, where, $\mu_{b}$ is the membership value for $b$. The property large corresponds to the value greater than $\mathrm{b}$ with a membership value $\left\{\mu_{b}<\mu \leq\right.$ $1\}$.

The next step is the penalization of pixels for which edges are not detected in the considered nearest neighborhood window. The following rule is used for penalization :

if $\nabla_{F}^{k}(i, j) \hat{n}$ is small, then $\Delta^{k}(i, j) \hat{n}=\nabla^{k}(i, j) \hat{n}$.

$$
\text { else, } \Delta^{k}(i, j) \hat{n}=0
$$

where, $\Delta^{k}(i, j) \hat{n}$ is the feedback at site $(i, j)$ due to the adjacent pixel in the direction $\hat{n}$. Eight such rules are used to get the contribution from all the eight directions. Hence, the total correction term $\Delta_{T}^{k}(i, j)$ for pixel at $(i, j)$ considering all the directions is given by,

$$
\Delta_{T}^{k}(i, j)=\frac{1}{8} \sum_{\hat{n}} \Delta^{k}(i, j) \hat{n}
$$

Replacing the error term $\sum_{j \in N_{i}}\left(\frac{\partial V\left(\lambda_{i}, \lambda_{j}\right)}{\partial \lambda_{i}}\right)_{\lambda_{i}=\lambda_{i}^{k}}$ in eqn.(6) by $\Delta_{T}^{k}(i)$, the OSL-algorithm modifies to,

$$
\lambda_{i^{\prime}}^{k+1}=\frac{\lambda_{i^{\prime}}^{k}}{\left[\sum_{j=1}^{M} p_{i^{\prime} j}+\frac{1}{\beta} \Delta_{T}^{k}\left(i^{\prime}\right)\right]} \sum_{j=1}^{M} \frac{y_{j} p_{i^{\prime} j}}{\sum_{o=1}^{N} \lambda_{o}^{k} p_{o j}}
$$

where, coordinates $(i, j)$ is denoted by a single coordinate $\left\{i^{\prime}=(i-1) * \sqrt{N}+j\right\}$. In the iterative image reconstruction procedure, the final correction term is fed back to update the 
pixel after each iteration. The iterations are continued until acceptable convergence is obtained.

Above fuzzy rules are also extended for $5 \times 5$ neighborhood window to study the effect of window size on the image quality. In the case of $3 \times 3$ window the fuzzy directional derivative is calculated using three elementary derivatives per direction. The sensitivity of edge detection depends upon the number of derivatives used for edge detection. To enhance the detectivity of edges, five elementary derivatives per direction are taken and 3:5 rule is adopted for edge detection.

\section{Simulated ExPerimental Results}

\section{A. Simulated PET System}

The algorithm was tested on a simulated PET system. The PET system consists of a ring detector with 64 detectors and the object space is decomposed into $64 \times 64$ square pixels. The object space is a square region inscribed within the circle of detectors. Each element of the probability matrix $p_{i j}$ defines the probability of a photon getting detected in the detector $j$ after emanating from the object pixel $i$. For simplicity, we assumed that $p_{i j}$ depends only on the geometry of the measurement system. This is taken as the angle $\theta_{i j}$ seen by the center of the pixel $i$ into the detector tube $j$ [1] i.e, $p_{i j}=\frac{\theta_{i j}}{\pi}$. Before the reconstruction begins, the probability matrix $\mathbf{P}=\left[p_{i j}\right], i=1, \ldots, N$ and $j=1, \ldots, M$ is computed and stored. For simulating measurement data, a Monte Carlo procedure is used [1][11]. We have used a source image with 100,000 counts.

\section{B. Algorithm Evaluation}

All the evaluation tests defined in this section are carried out on a simulated PET system. The proposed algorithm with $3 \times 3$ and $5 \times 5$ neighborhood window are used. For compact representation in the rest of the paper, proposed algorithm with $3 \times 3$ and $5 \times 5$ neighborhood window are named as F1 and F2 algorithm respectively. The results are also compared with MAP reconstruction algorithm. MAP with potential $V\left(\lambda_{i}-\right.$ $\left.\lambda_{j}\right)=\sum_{j \in N_{i}}\left(\lambda_{i}=\lambda_{j}\right)^{2}$ and $\beta=2.5 \times 10^{4}$ is used in the present study. This choice of $\beta$ has produced best estimate and hence it is considered. The performances of the proposed new algorithm are evaluated using three different quantitative test as given below :

1) Log-likelihood Test: All the algorithms described in section II and III compute the estimate of the emission densities iteratively, hence log-likelihood function is an appropriate qualitative measure. For an estimate $\lambda^{k}$, the log-likelihood function $l\left(\lambda^{k}\right)$ at $k^{t h}$ iteration is defined as,

$$
l\left(\lambda^{k}\right)=\sum_{j=1}^{M}\left[-\phi_{j}^{k}+y_{j} \log \phi_{j}^{k}-\log \left(y_{j} !\right)\right]
$$

where, $\phi_{j}^{k}=\sum_{i=1}^{N} \lambda_{i}^{k} p_{i j}$ is the pseudo-projection in the tube $j$.

The log-likelihood values of the reconstructed images obtained using MAP, F1 and F2 algorithms are plotted against the number of iterations in Fig.2. It is clearly evident that

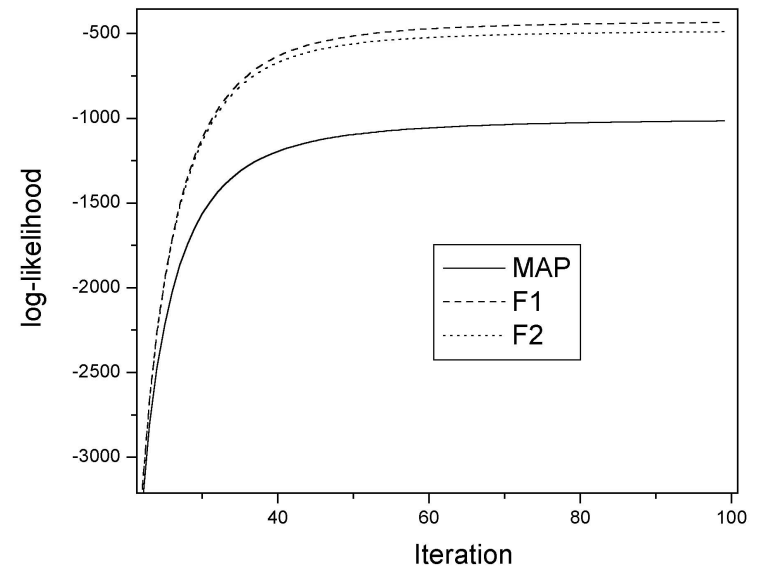

Fig. 2. Log-likelihood values for MAP, F1 and F2 algorithms.

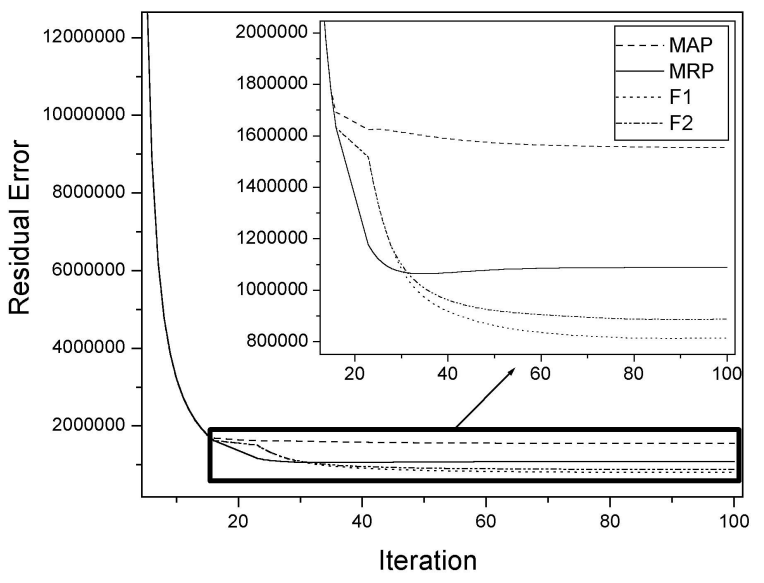

Fig. 3. Residual error versus iteration plot for MAP, MRP, F1 and F2 algorithms.

log-likelihood for the proposed algorithm converges faster compared to MAP-algorithm.

2) Residual Error: In iterative image reconstruction techniques, residual error is the most prefered evaluation test. This measures the deviation of the generated pseudo-projections $\phi_{j}^{k}$ of the reconstructed image from the observed projection data $y_{j}$. Residual error $\rho\left(\lambda^{k}\right)$ at $k^{t h}$-iteration is given by,

$$
\rho\left(\lambda^{k}\right)=\sum_{j=1}^{M}\left(y_{j}-\phi_{j}^{k}\right)^{2}
$$

In Fig.3, the residual errors of the reconstructed images for the proposed F1 and F2 algorithms along with MAP and MRP algorithms are shown. From this plots it is clear that proposed algorithm has the lowest residual error compared to the MAP 


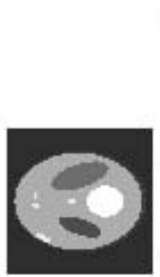

(a)

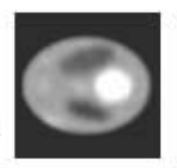

(b)

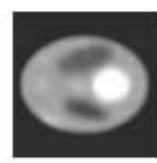

(f)

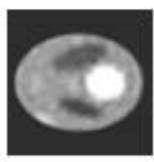

(c)

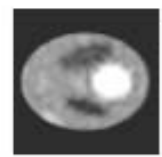

(g)

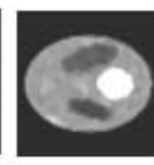

(d)

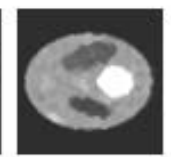

(h)

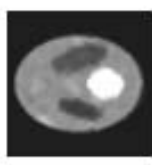

(e)

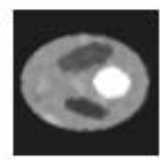

(i)
Fig. 4. (a) Original test phantom, (b),(c),(d),(e) and (f),(g),(h),(i) are the reconstruction using MAP, MRP, F1, F2 algorithms after 50 and 100 iterations respectively.

and MRP algorithms used in this evaluation.

3) Visual Inspection: In, this section, qualitatively comparisons are made from the results of the reconstruction algorithms. In Fig.4, (b,c,d,e) and (f,g,h,i) show the reconstructed images using MAP, MRP, F1, F2 after 50 and 100 iterations respectively. For quality assessment, the original test image(phantom) is also shown (see Fig.4(a)). The images reconstructed using the proposed algorithm (see Fig.4 (d),(e),(h) and (i)) are more appealing and rich in edges. The proposed fuzzy algorithm compares favorably with the MAP and MRP algorithms.

\section{CONCLUSION}

A new approach is presented towards edge preserving reconstruction of the emission densities for ET, based on the application of fuzzy rule based techniques to model the potential (which accounts for the nearest neighbor interaction) in image reconstruction problem. Two basic operations are performed namely, fuzzy filtering and fuzzy smoothing. Fuzzy filtering is used for the detection of edges in the reconstruction while fuzzy smoothing is used to penalize only those pixels for which the edges are absent in the nearest neighborhood. This dual operation is continued iteratively until acceptable convergence is obtained. Computer simulated experimental PET studies reveal the superiority of the proposed algorithm over the existing algorithms like MAP and MRP. It is found that residual error is low for the proposed algorithm. Visual representation of the reconstructed images using proposed algorithms are found better than MAP and MRP reconstructed images.

\section{ACKNOWLEDGMENT}

The first author would like to thank Council of Scientific and Industrial research (CSIR), Government of India, for providing Junior Research fellowship. He declares that this work is partially supported by CSIR, New Delhi, India.

\section{REFERENCES}

[1] L.A. Shepp and Y. Vardi., "Maximum likelihood estimation for emission tomography ", IEEE Trans. on Medical Imaging ., MI-1: pp. 113-121, 1982.

[2] P.J. Green, "Bayesian reconstruction from emission tomography data using a modified EM algorithm", IEEE Trans. on Med. Img., vol.9, No.1, March, 1990.

[3] Z. Zhou, R. M. Leahy and J. Qi, " Approximate maximum likelihood hyperparameter estimation for Gibbs prior ", IEEE Trans. on Img. proc., Vol.6, No.6, pp.844-861, June, 1997.

[4] J. Nuyts, D. Bequ, P. Dupont, and L. Mortelmans, "A Concave Prior Penalizing Relative Differences for Maximum-a-Posteriori Reconstruction in Emission Tomography ", IEEE Trans. on Nuclear Science, Vol. 49, No. 1, pp.56-60, Feb. 2002

[5] S. Alenius and U. Ruotsalainen, "Using Local Median as the Location of Prior Distribution in Iterative Emission Tomography Reconstruction ", IEEE Tran. Nucl. Sci., Vol.45, No.6, Dec. 1998.

[6] S. Alenius and U. Ruotsalainen, "Generalization of Median Root Prior Reconstruction ”, IEEE Trans. Med. Img., Vol.21, No.11, Nov., 2002.

[7] J. Besag, "Spatial interaction and the statistical analysis of lattice systems ", Jl. of Royal Stat. Soc. B, Vol.36, pp. 192-236, 1974.

[8] D. V. Ville, M. Nachtegael, D. V. Weken, E. E. Kerre, W. Philips and I. Lemahieu, “ Noise Reduction by Fuzzy Image Filtering " ,IEEE Trans. Fuzzy Sys., Vol. 11, NO. 4, Aug. 2003.

[9] L. A. Zadeh, "Fuzzy Logic", IEEE Computer, pp.83-93, April 1988.

[10] S. Geman and D.Geman, "Stocastic relaxation, Gibbs distribution and the Bayesian restoration of images ", IEEE Trans. Pattern Anal. Machine Intell., vol. PAMI-6, pp. 721-741, Nov., 1984.

[11] N. Rajeevan, K. Rajgopal and G. Krishna. " Vector-Extrapolated fast maximum likelihood estimation algorithms for emission tomography ", IEEE Trans. on Med. Img., Vol. 11, No.1, March 1992. 